\title{
EZH2 mutations in chronic myelomonocytic leukemia cluster with ASXL1 mutations and their co-occurrence is prognostically detrimental
}

\author{
Mrinal M. Patnaik (1) ', Rangit Vallapureddy ${ }^{1}$, Terra L. Lasho ${ }^{1}$, Katherine P. Hoversten ${ }^{1}$, Christy M. Finke ${ }^{1}$, Rhett Ketterling ${ }^{2}$, \\ Curtis Hanson ${ }^{2}$, Naseema Gangat ${ }^{1}$ and Ayalew Tefferi ${ }^{1}$
}

Chronic myelomonocytic leukemia (CMML) is an aggressive hematological malignancy characterized by sustained peripheral blood monocytosis and an inherent risk for leukemic blast transformation ${ }^{1,2}$. Patients with CMML have between 10-15 mutations per kilobase of coding DNA regions; with majority of these (>90\%) involving epigenetic regulator genes (TET2 60\%, ASXL1 $40 \%$ ), splicing machinery (SRSF2 40\%), and cell signaling (oncogenic $R A S$ pathway $30 \%)^{3-5}$. The polycomb group proteins play an important role in transcriptional repression by regulating chromatin modifications and consist of two canonical polycomb repressive complexes (PRC) PRC1 and PRC2 ${ }^{6}$. PRC2, comprises EED (embryonic ectoderm development protein), EZH2 (enhancer of zeste homolog 2), SUZ12 (suppressor of zeste 12 protein homolog), and RBBP4/7 (retinoblastoma binding protein), is recruited to chromatin and results in the trimethylation of lysine 27 of the histone 3 mark (H3K27me3), a repressive mark silencing gene transcription. In addition, ASXL1 (additional sex combs like 1) has been shown to associate with $\mathrm{PRC} 2$ and results in global reductions in $\mathrm{H} 3 \mathrm{~K} 27 \mathrm{me} 3$, suggesting a regulatory role in the $\mathrm{PRC} 2^{7}$.

ASXL1 mutations (frameshift and nonsense) result in the truncation of the ASXL1 protein, are seen in $~ 40 \%$ of CMML patients, and independently and adversely impact overall survival $(\mathrm{OS})^{4,8}$. EZH2 mutations (chromosome 7q36.1) in CMML, unlike in epithelial malignancies and

\footnotetext{
Correspondence: Mrinal M. Patnaik (Patnaik.mrinal@mayo.edu)

'Division of Hematology, Department of Internal Medicine, Mayo Clinic, Rochester, MN, USA

²Department of Laboratory Medicine and Pathology, Mayo Clinic, Rochester,
} MN, USA lymphoproliferative disorders, are loss-of-function mutations and are uncommon $(<5 \%)$, with an indeterminate prognostic impact ${ }^{4,9}$. Given that the co-occurrence of these mutations have been documented in myeloid neoplasms and that theoretically these could further impact the repressive role of the PRC2, we examined a large and informative CMML data set to assess the prognostic impact of $A S X L 1$ and $E Z H 2$ co-mutations in $\mathrm{CMML}^{10}$.

Patients with 2016 WHO (World Health Organization)defined CMML were identified from the institutional database $^{1}$. Al.(BM) biopsies and cytogenetic studies performed at diagnosis. A 29 gene panel next-generation sequencing assay was carried out on BM DNA specimens on all 277 patients obtained at diagnosis for the following genes: TET2, DNMT3A, IDH1, IDH2, ASXL1, EZH2, SUZ12, SRSF2, SF3B1, ZRSR2, U2AF1, PTPN11, Tp53, SH2B3, RUNX1, CBL, NRAS, KRAS, JAK2, CSF3R, FLT3, KIT, CALR, MPL, NPM1, CEBPA, IKZF, ETNK1, and SETBP1, by previously described methods ${ }^{5,9}$. All statistical analyses considered parameters obtained at time of CMML diagnosis. Differences in the distribution of continuous variables between categories were analyzed by either Mann-Whitney or Kruskal-Wallis tests. Patient groups with nominal variables were compared by $\chi^{2}$ test. OS was calculated from the date of first referral to date of death or last contact. Leukemia-free survival (LFS) was calculated from the date of first referral to date of leukemic transformation or death/last contact. Overall and LFS curves were prepared by the Kaplan-Meier method and compared by the log-rank test. Cox proportional hazard regression model was used for multivariable analysis. 
Table 1 Clinical and laboratory characteristics of 277 CMML patients stratified by their ASXL1 and EZH2 mutational status

\begin{tabular}{|c|c|c|c|c|c|}
\hline Variables & $\begin{array}{l}\text { All patients with } \\
\text { CMML }(n=277)\end{array}$ & $\begin{array}{l}\text { ASXL1mt/EZH2mt } \\
\text { CMML patients }(n= \\
7,3 \%)\end{array}$ & $\begin{array}{l}\text { ASXL1mt CMML } \\
\text { patients ( } n=131, \\
47 \%)\end{array}$ & $\begin{array}{l}\text { ASXL1wt CMML } \\
\text { patients ( } n=139 \text {, } \\
50 \%)\end{array}$ & $\begin{array}{l}\text { P-value comparing } A S X L 1 m t / \\
E Z H 2 m t \text { patients with } \\
\text { ASXL1mt and } A S X L 1 w t\end{array}$ \\
\hline Age in years; median (range) & $72.3(18-92)$ & $67(65-79)$ & $72.5(27-92)$ & $72.4(18-92)$ & 0.7 \\
\hline Males; $n(\%)$ & $183(66)$ & $6(86)$ & $89(68)$ & $88(63)$ & 0.4 \\
\hline $\begin{array}{l}\text { Hemoglobin, } \mathrm{g} / \mathrm{dL} \text {; median } \\
\text { (range) }\end{array}$ & $10.7(6.4-17)$ & $11.3(7.2-15)$ & $10.7(6.4-16.8)$ & $10.9(7.1-17)$ & 0.7 \\
\hline WBC $\times 10^{9} / L ;$ median (range) & $12.3(2-265)$ & $14.1(6-51)$ & $14.3(2-265)$ & $10(2-186)$ & 0.01 \\
\hline ANC $\times 10^{9} / \mathrm{L} ;$ median (range) & $6.2(0.1-151$ & $6.3(3.2-32)$ & $7.6(0.2-151)$ & $5.2(0.1-143)$ & 0.04 \\
\hline AMC $\times 10^{9} / \mathrm{L} ;$ median (range) & $2.5(1-40)$ & $2.8(1-8)$ & $3(1-40)$ & $2(1-30)$ & 0.007 \\
\hline ALC $\times 10^{9} / \mathrm{L} ;$ median (range) & $1.7(0-22)$ & $1.6(1.2-6)$ & $1.9(0.4-22)$ & $1.6(0-11)$ & 0.13 \\
\hline $\begin{array}{l}\text { Platelets } \times 10^{9} / \mathrm{L} \text {; median } \\
\text { (range) }\end{array}$ & $98(10-840)$ & $211(25-526)$ & $95(10-726)$ & $100(12-840)$ & 0.1 \\
\hline $\begin{array}{l}\text { Presence of circulating } \\
\text { immature myeloid cells; } n \\
\text { (\%) }\end{array}$ & $156(56)$ & $5(71)$ & $81(62)$ & $70(50)$ & 0.09 \\
\hline PB blast \%; median (range) & $0(0-19)$ & $0(0-4)$ & $0(0-19)$ & $0(0-12)$ & 0.6 \\
\hline BM blast \%; median (range) & $3(0-19)$ & $1(0-10)$ & $4(0-19)$ & $3(0-18)$ & 0.08 \\
\hline $\begin{array}{l}\text { Lactate dehydrogenase } \\
\text { levels IU/ml; median (range) }\end{array}$ & $226(84-1296)$ & $271(163-604)$ & $243(84-1296)$ & $215(131-719)$ & 0.1 \\
\hline Cytogenetics & $(n=267)$ & & $(n=124)$ & $(n=136)$ & 0.7 \\
\hline abnormal; n (\%) & $85(32)$ & $3(43)$ & $41(33)$ & $41(30)$ & \\
\hline FAB CMML classification & $(n=276)$ & & & $(n=138)$ & 0.005 \\
\hline Proliferative & $135(49)$ & $4(57)$ & $77(59)$ & $54(39)$ & \\
\hline Dysplastic & $141(51)$ & $3(43)$ & $54(41)$ & $84(61)$ & \\
\hline Therapy-related CMML; n (\%) & $28(10$ & $1(14)$ & $11(8)$ & $16(12)$ & 0.7 \\
\hline \multicolumn{6}{|c|}{ Next-generation sequencing analysis; $n$ (\%) } \\
\hline \multicolumn{6}{|l|}{ 1. Epigenetic regulators } \\
\hline TET2 & $154(56)$ & $4(57)$ & $59(45)$ & $91(65)$ & 0.003 \\
\hline DNMT3A & $16(6)$ & 0 & $7(5)$ & $9(6)$ & 0.7 \\
\hline $\mathrm{IDH} 1$ & $5(2)$ & 0 & $3(2)$ & $2(1)$ & 0.8 \\
\hline $\mathrm{IDH} 2$ & $16(6)$ & 0 & $8(6)$ & $8(6)$ & 0.8 \\
\hline \multicolumn{6}{|l|}{ 2. Transcription factors } \\
\hline RUNX1 & $21(8)$ & $3(43)$ & $11(8)$ & $7(5)$ & 0.001 \\
\hline$B C O R$ & $1(0.5)$ & $1(14)$ & 0 & 0 & $<0.0001$ \\
\hline \multicolumn{6}{|l|}{ 3. Spliceosome components } \\
\hline SF3B1 & $15(5)$ & 0 & $1(1)$ & $14(10)$ & 0.003 \\
\hline SRSF2 & $129(47)$ & $2(29)$ & $63(48)$ & $64(46)$ & 0.6 \\
\hline U2AF1 & $19(7)$ & 0 & $12(9)$ & $7(5)$ & 0.3 \\
\hline ZRSR2 & $8(3)$ & 0 & $2(2)$ & $6(4)$ & 0.4 \\
\hline
\end{tabular}


Table 1 continued

\begin{tabular}{|c|c|c|c|c|c|}
\hline Variables & $\begin{array}{l}\text { All patients with } \\
\text { CMML }(n=277)\end{array}$ & $\begin{array}{l}\text { ASXL1mt/EZH2mt } \\
\text { CMML patients }(n= \\
7,3 \%)\end{array}$ & $\begin{array}{l}\text { ASXL1mt CMML } \\
\text { patients ( } n=131 \text {, } \\
47 \%)\end{array}$ & $\begin{array}{l}\text { ASXL1wt CMML } \\
\text { patients ( } n=139 \text {, } \\
50 \%)\end{array}$ & $\begin{array}{l}P \text {-value comparing } A S X L 1 m t / \\
E Z H 2 m t \text { patients with } \\
\text { ASXL1mt and } A S X L 1 w t\end{array}$ \\
\hline \multicolumn{6}{|l|}{ 4. Cell signaling } \\
\hline JAK2 V617F & $20(7)$ & $1(14)$ & $9(7)$ & $10(7)$ & 0.8 \\
\hline$M P L$ & $2(1)$ & 0 & 0 & $2(1)$ & 0.4 \\
\hline $\mathrm{SH} 2 \mathrm{~B} 3$ & $1(0.5)$ & 0 & $1(1)$ & 0 & 0.6 \\
\hline$C B L$ & $37(13)$ & 0 & $20(15)$ & $17(12)$ & 0.4 \\
\hline NRAS & $44(16)$ & $1(14)$ & $25(19)$ & $18(13)$ & 0.4 \\
\hline KRAS & $12(4)$ & $1(14)$ & $8(6)$ & $3(2)$ & 0.1 \\
\hline PTPN11 & $8(3)$ & 0 & $5(4)$ & $3(2)$ & 0.6 \\
\hline CSF3R & $4(1)$ & 0 & $2(2)$ & $2(1)$ & 0.9 \\
\hline$C-K I T$ & $8(3)$ & 0 & $4(3)$ & $4(3)$ & 0.9 \\
\hline FLT3 & $7(3)$ & $1(14)$ & $1(1)$ & $5(4)$ & 0.04 \\
\hline NPM1 & $1(0.5)$ & 0 & 0 & $1(1)$ & 0.6 \\
\hline CALR & $1(0.5)$ & 0 & 0 & $1(1)$ & 0.6 \\
\hline \multicolumn{6}{|l|}{ 5. Tumor suppressor genes } \\
\hline $\operatorname{Tp53}$ & $7(3)$ & 0 & $1(1)$ & $6(4)$ & 0.2 \\
\hline \multicolumn{6}{|l|}{ 6. Others } \\
\hline SETBP1 & $37(13)$ & $1(14)$ & $26(20)$ & $10(7)$ & 0.009 \\
\hline CEBPA & $3(1)$ & 0 & $1(1)$ & $2(1)$ & 0.8 \\
\hline $\begin{array}{l}2016 \text { WHO morphological } \\
\text { subtypes; } n(\%)\end{array}$ & $(n=275)$ & & $(n=129)$ & & 0.9 \\
\hline CMML-0 & $155(56)$ & $5(71)$ & $73(57)$ & $77(55)$ & \\
\hline CMML-1 & $70(25)$ & $1(14)$ & $31(24)$ & $38(27)$ & \\
\hline CMML-2 & $50(18)$ & $1(14)$ & $25(19)$ & $24(17)$ & \\
\hline $\begin{array}{l}\text { Spanish cytogenetic risk } \\
\text { stratification; } n(\%)\end{array}$ & $(n=270)$ & & $(n=126)$ & $(n=137)$ & 0.5 \\
\hline Low & $202(75)$ & $4(57)$ & $90(71)$ & $108(79)$ & \\
\hline Intermediate & $42(16)$ & $2(29)$ & $22(17)$ & $18(13)$ & \\
\hline High & $26(10)$ & $1(14)$ & $14(11)$ & $11(18)$ & \\
\hline $\begin{array}{l}\text { Mayo-French cytogenetic } \\
\text { risk stratification; } n(\%)\end{array}$ & $(n=270)$ & & $(n=126)$ & $(n=137)$ & 0.2 \\
\hline Low & $201(74)$ & $4(57)$ & $89(71)$ & $108(79)$ & \\
\hline Intermediate & $55(20)$ & $2(29)$ & $32(25)$ & $21(15)$ & \\
\hline High & $14(5)$ & $1(14)$ & $5(4)$ & $8(6)$ & \\
\hline $\begin{array}{l}\text { Mayo prognostic model; } n \\
\text { (\%) }\end{array}$ & $(n=274)$ & & $(n=129)$ & $(n=138)$ & 0.14 \\
\hline Low & $86(31)$ & 0 & $35(27)$ & $51(37)$ & \\
\hline Intermediate & $88(32)$ & $4(57)$ & $45(35)$ & $39(28)$ & \\
\hline High & $100(36)$ & $3(43)$ & 49 (38) & $48(35)$ & \\
\hline
\end{tabular}


Table 1 continued

\begin{tabular}{|c|c|c|c|c|c|}
\hline Variables & $\begin{array}{l}\text { All patients with } \\
\text { CMML }(n=277)\end{array}$ & $\begin{array}{l}\text { ASXL1mt/EZH2mt } \\
\text { CMML patients }(n= \\
7,3 \%)\end{array}$ & $\begin{array}{l}\text { ASXL1mt CMML } \\
\text { patients }(n=131, \\
47 \%)\end{array}$ & $\begin{array}{l}\text { ASXL1wt CMML } \\
\text { patients ( } n=139 \text {, } \\
50 \%)\end{array}$ & $\begin{array}{l}P \text {-value comparing } A S X L 1 m t / \\
E Z H 2 m t \text { patients with } \\
\text { ASXL1mt and } A S X L 1 w t\end{array}$ \\
\hline Molecular Mayo model; $n$ (\%) & $(n=260)$ & & $(n=126)$ & $(n=127)$ & $<0.0001$ \\
\hline Low & $20(8)$ & 0 & 0 & $20(16)$ & \\
\hline Intermediate-1 & $67(26)$ & 0 & $19(15)$ & $48(38)$ & \\
\hline Intermediate-2 & $83(32)$ & $4(57)$ & $37(29)$ & $42(33)$ & \\
\hline High & $90(35)$ & $3(43)$ & $70(56)$ & $17(13)$ & \\
\hline $\begin{array}{l}\text { GFM CMML prognostic } \\
\text { model; } n(\%)\end{array}$ & $(n=268)$ & & $(n=129)$ & $(n=132)$ & $<0.0001$ \\
\hline Low & $117(44)$ & $1(14)$ & $29(22)$ & $87(66)$ & \\
\hline Intermediate & $100(37)$ & $4(57)$ & $59(46)$ & $37(28)$ & \\
\hline High & $51(19)$ & $2(29)$ & $41(32)$ & $8(6)$ & \\
\hline $\begin{array}{l}\text { Leukemic transformation; } n \\
\text { (\%) }\end{array}$ & $48(17)$ & 0 & $27(21)$ & $21(15)$ & 0.23 \\
\hline Deaths; $n$ (\%) & $169(61)$ & $3(43)$ & $87(66)$ & $79(57)$ & 0.2 \\
\hline $\begin{array}{l}\text { Follow-up in months; } \\
\text { median (range) }\end{array}$ & $16(0.03-194)$ & $6(0.2-24) 0.07-$ & $15(0.07-183)$ & $19(0.03-194)$ & 0.08 \\
\hline
\end{tabular}

The bold values represent statistically significant $p$ values, $p<0.05$

$m t$ mutant, wt wild type, $W B C$ white blood cell count, $A N C$ absolute neutrophil count, $A M C$ absolute monocyte count, $A L C$ absolute lymphocyte count, $P B$ peripheral blood, $B M$ bone marrow, WHO World Health Organization, GFMGroupe Francophone des Myélodysplasies, FABFrench-American-British, $B T$ blast transformation

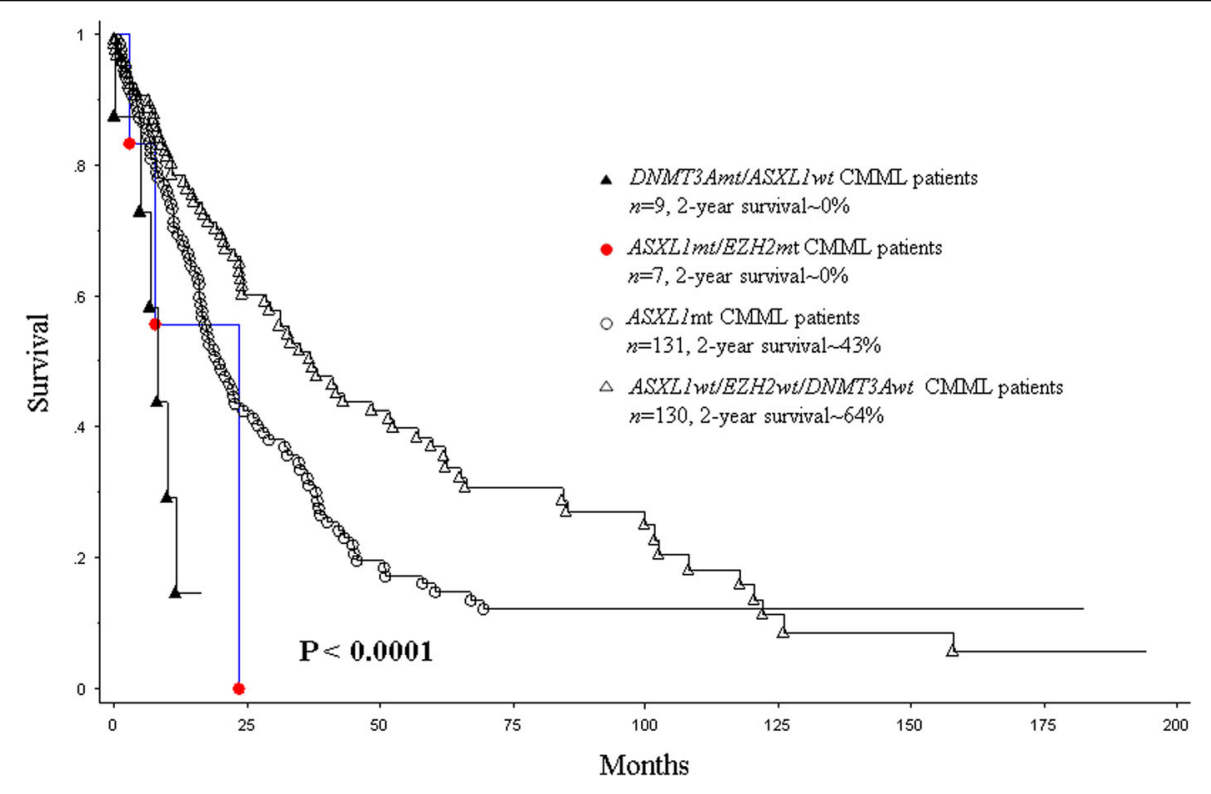

Fig. 1 Overall survival of $A S X L 1, E Z H 2$, and DNMT3A mutational status

Two hundred and seventy-seven WHO-defined CMML patients were included in the study; median age 72 years (range, 18-92), 66\% males. ASXL1 mutations were identified in $138(50 \%)$ patients, whereas $E Z H 2$ mutations were identified in 7 (3\%) patients; all 7 (100\%) being co- mutated for ASXL1 (Table 1). EZH2 mutation types included; missense 3 (43\%), nonsense 2 (28\%), and 1 (14\%) each for frameshift mutations and intronic mutations impacting splicing (splice site mutation). Four (57\%) patients with $E Z H 2$ mutations had a proliferative CMML 
phenotype, while three (43\%) patients had an abnormal karyotype, including trisomy 8 , isochromosome $17 \mathrm{q}$, and a balanced translocation- $t(3 ; 12 ; 6)(\mathrm{p} 21 ; \mathrm{q} 21 ; \mathrm{q} 23)$, respectively. Mutational frequencies in the ASXL1/EZH2 comutated cohort included TET2 57\%, RUNX1 43\%, SRSF2 $29 \%$, and $14 \%$ each for JAK2V617F, FLT3-ITD, BCOR, $C B L, N R A S$, and SETBP1. Notably there were no mutations involving DNMT3A, IDH1, IDH2, SF3B1, U2AF1, ZRSR2, or TP53. In comparison to ASXL1mut/EZH2wt and $A S X L 1 \mathrm{wt} / E Z H 2 \mathrm{wt}$, patients with $A S X L 1 / E Z H 2$ comutations were more likely to have additional mutations involving RUNX1 (43\%, $p=0.001), B C O R(14 \%, p<$ $0.0001), F L T 3-I T D(14 \%, p=0.04)$ and less likely to have SF3B1 mutations $(0 \%, p=0.003)$. The ASXL1/EZH2 comutated patients were also more likely to have higher risk stratification by the ASXL1 integrated Mayo Molecular Model $(p<0.0001)$ and the GFM CMML prognostic model $(p<0.0001)$.

At last follow-up (median, 16 months), 169 (61\%) deaths and 48 (17\%) leukemic transformations were documented. Median survival for ASXL1/EZH2 comutated patients was 16 months, in comparison to 20 months for $A S X L 1 \mathrm{mt} / E Z H 2 \mathrm{wt}$ and 33 months for $A S X L 1 \mathrm{wt} / E Z H 2 \mathrm{wt}$ patients $(p<0.0001$, Fig. 1$)$. In a univariate analysis, survival (OS) was adversely impacted by male sex $(p=0.03)$, low hemoglobin $(\mathrm{HB}<10 \mathrm{gm} / \mathrm{dl}, p=$ 0.001 ), high white blood cell count (WBC $>15 \times 10(9) / \mathrm{L}$; $p=0.0003$ ), high absolute monocyte count (AMC $>10 \times$ $10(9) / \mathrm{L}, p=0.0002)$, high absolute lymphocyte count ( $p$ $=0.02$ ), presence of circulating immature myeloid cells (IMC, $p=0.002)$, peripheral blood $(p=0.001)$ and $\mathrm{BM}(p$ $=0.045)$ blast $\%$, abnormal karyotype $(p=0.0008)$, absence of TET2 mutations $(p=0.0003)$, presence of $\operatorname{ASXL1}(p=0.009), D N M T 3 A(p=0.001)$, and Tp53 $(p=$ $0.02)$ mutations. $E Z H 2$ mutations by themselves did not impact $\operatorname{OS}(p=0.2)$; however, when analyzed in the context of $A S X L 1 \mathrm{mt} / E Z H 2 \mathrm{mt}$ status, in comparison to ASXL1mutations, the adverse impact of the co-mutations was significantly stronger $(p=0.04$, HR $2.9,95 \%$ CI 1.1-9.5). In a multivariable survival analysis that included the aforementioned significant variables, only high WBC $>15 \times 10(9) / \mathrm{L} \quad(p=0.005, \quad$ HR $\quad 1.005, \quad 95 \% \quad$ CI 1.003-1.009), male sex $(p=0.002$, HR $1.7,95 \%$ CI $1.2-2.4)$, presence of IMC $(p=0.009$, HR $1.5,95 \%$ CI 1.1-2.4), presence of ASXL1/EZH2 co-mutations ( $p=$ 0.03, HR $2.195 \%$ CI 1.2-3.2), presence of DNMT3A mutations $(p=0.002$, HR 2.8, 95\% CI 1.4-5.4), and absence of TET2 mutations ( $p=0.0006$, HR $1.7,95 \%$ CI 1.2-2.4) independently and adversely impacted OS. The prognostic relevance of $A S X L 1 / E Z H 2$ co-mutational status was lost when assessed in context of the Mayo Molecular Model $(p=0.2)$ and the GFM CMML model $(p=0.4)$. Neither did ASXL1 $(p=0.14)$ nor EZH2 $(p=$ 0.3) mutations impact LFS.
In summary, our study has demonstrated that $E Z H 2$ mutations are infrequent $(<5 \%)$ in WHO-defined CMML, almost always co-occur with $A S X L 1$ mutations, are not associated with $D N M T 3 A$ or SF3B1 mutations, and are frequently associated with a "proliferative" CMML phenotype. While $E Z H 2$ mutations themselves did not impact either OS or LFS, ASXL1/EZH2 co-mutated patients had higher risk stratification by the $A S X L 1$-integrated CMML prognostic models and had a shorter survival, in comparison to ASXL1mt patients alone. Mechanistic studies, including chromatin immunoprecipitation and sequencing (ChIP-seq) are needed to see if the ASXL1/EZH2 comutational status indeed synergistically impacts PRC2 activity and further depletes methylation of H3K27, resulting in unbridled transcription.

\section{Acknowledgements}

Current publication is supported in part by grants from the "The Gerstner Family Career Development Award" and the "Mayo Clinic Center for Individualized Medicine, Mayo Clinic, Rochester, MN, USA". This publication was supported by CTSA Grant Number KL2 TR000136 from the National Center for Advancing Translational Science (NCATS). Its contents are solely the responsibility of the authors and do not necessarily represent the official views of the $\mathrm{NIH}$.

\section{Conflict of interest}

The authors declare that they have no conflict of interest.

\section{Publisher's note}

Springer Nature remains neutral with regard to jurisdictional claims in published maps and institutional affiliations.

Received: 15 November 2017 Accepted: 29 November 2017 Published online: 22 January 2018

\section{References}

1. Arber, D. A. et al. The 2016 revision to the World Health Organization classification of myeloid neoplasms and acute leukemia. Blood 127, 2391-2405 (2016).

2. Patnaik, M. M. \& Tefferi, A. Chronic myelomonocytic leukemia: 2016 update on diagnosis, risk stratification, and management. Am. J. Hematol. 91, 631-642 (2016).

3. Merlevede, J. et al. Mutation allele burden remains unchanged in chronic myelomonocytic leukaemia responding to hypomethylating agents. Nat. Commun. 7, 10767 (2016).

4. Patnaik, M. M. et al. ASXL1 and SETBP1 mutations and their prognostic contribution in chronic myelomonocytic leukemia: a two-center study of 466 patients. Leukemia 28, 2206-2212 (2014).

5. Patnaik, M. M. et al. Prognostic interaction between ASXL1 and TET2 mutations in chronic myelomonocytic leukemia. Blood Cancer J. 6, e385 (2016).

6. Iwama, A. Polycomb repressive complexes in hematological malignancies. Blood 130, 23-29 (2017).

7. Abdel-Wahab, O. et al. ASXL1 mutations promote myeloid transformation through loss of PRC2-mediated gene repression. Cancer Cell. 22, 180-193 (2012).

8. Itzykson, R. et al. Prognostic score including gene mutations in chronic myelomonocytic leukemia. J. Clin. Oncol. 31, 2428-2436 (2013).

9. Patnaik, M. M. et al. DNMT3A mutations are associated with inferior overall and leukemia-free survival in chronic myelomonocytic leukemia. Am. J. Hematol. 92, 56-61 (2017).

10. Abdel-Wahab, O. et al. Concomitant analysis of EZH2 and ASXL1 mutations in myelofibrosis, chronic myelomonocytic leukemia and blast-phase myeloproliferative neoplasms. Leukemia 25, 1200-1202 (2011). 